\title{
Surgical treatment of the rheumatoid hip
}

\author{
I G Kelly
}

Hip joint disease in rheumatoid arthritis is said to occur in between 30 and $40 \%$ of patients, ${ }^{12}$ higher figures being obtained in radiographically based studies. This is a considerably lower incidence of disease than for the knee $(60 \%)$ or shoulder (70\%), and the frequency with which the hip joint undergoes surgery is a direct result of the development of hip joint replacement surgery.

\section{Patterns of joint disease}

The inflammatory process within the hip joint initially leads to a synovitis with an effusion. To accommodate the increased volume the hip assumes a flexed, externally rotated, and adducted position. Fixed deformities quickly develop and are demonstrable in most patients at the time of presentation. Certain radiological patterns result from these pathological processes, ${ }^{2} 3$ the most common being concentric joint space loss, which may progress with erosion of the acetabular roof and, to a lesser extent, of the femoral head (fig 1). Between 15 and $20 \%$ of patients will develop protrusio acetabuli (fig 2) and without treatment this will

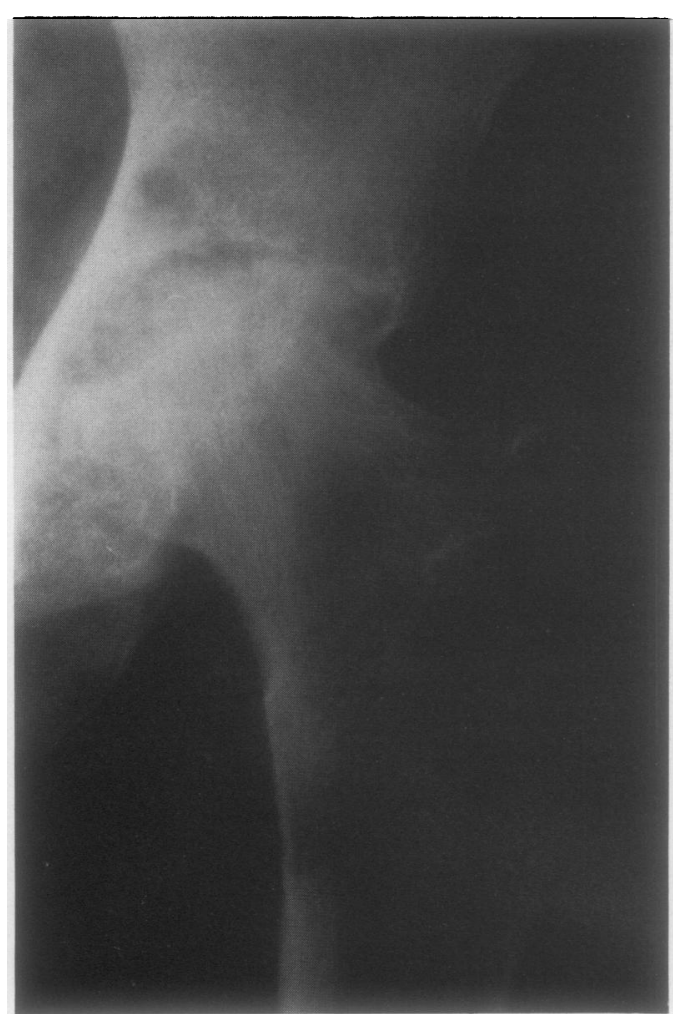

Figure 1 Typical concentric disease of the hip joint in rheumatoid arthritis, with erosion of both the femoral head I G Kelly and the acetabulum.

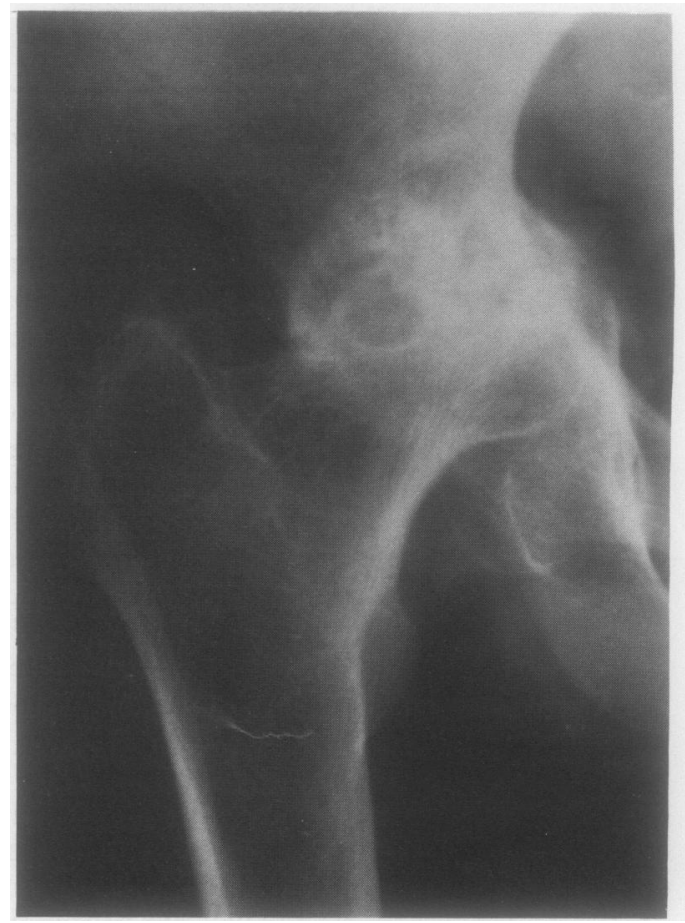

Figure 2 Protrusio acetabuli with fracture of the acetabular floor.

progress and may be complicated by fracture of the thin medial acetabular wall.

\section{Indications for surgery}

The patient's major complaint is pain, which is usually localised to the groin, buttock, or lateral thigh but is occasionally limited to the knee, causing diagnostic difficulties when the knee is also affected. If the pain cannot be controlled by analgesics or regularly disturbs sleep surgery is indicated. Stiffness or deformity can also provide an indication for surgery by threatening the patient's independence. Although it is well established that young patients have more problems with loosening of the prosthesis than older patients, ${ }^{4}$ in my practice youth is no contraindication if the symptoms cannot be relieved in any other way.

Disease of multiple joints of the leg is not uncommon, and in this situation a plan of management must be worked out with the patient and his/her family or carers. If surgery is to be carried out on all of the affected joints then it is my practice to start with the most painful. The hip will take precedence, however, if there is a rapidly progressing protrusio or if there is ipsilateral disease of the hip and knee. In this situation correction of the hip deformities 
facilitates the correct alignment of the knee arthroplasty.

\section{Preoperative assessment}

Before surgery, as with other major joint operations, the patient must be assessed for the presence of anaemia, impaired renal function, or any sites of active infection, such as unhealed skin lesions. All patients should also have lateral flexion and extension radiographs of their cervical spine carried out to detect any significant spinal disease (see p 846, this issue). The anaesthetist can then be alerted to its presence and appropriate measures can be taken for intubation, including the use of fibreoptic laryngoscopes. Radiological subluxation in the absence of symptoms or signs of a myelopathy does not constitute a contraindication to surgery.

\section{Surgical management}

Rheumatoid disease at most joints can be managed by any one of synovectomy, osteotomy, arthrodesis, or arthroplasty according to the stage of the arthritis. At the hip synovectomy is very difficult to perform adequately and has failed to give satisfactory results in the adult. ${ }^{5}$ It may have a role in juvenile chronic arthritis, but in this group its major effect is relief of pain with no significant improvement in mobility and, like synovectomy at most other sites, continued radiological progression. ${ }^{6}$ Osteotomies around the hip do nothing to tackle the rheumatoid pannus and are therefore inappropriate. Arthrodesis is contraindicated by the polyarticular nature and potential of the disease.

Total hip replacement is the most valuable form of surgical management for the rheumatoid hip. Developments over the last 30 years have resulted in a variety of hip-arthroplasties, all of which have a stemmed metal femoral component and a high molecular weight polyethylene acetabular component.

\section{Operative approaches}

Much of the fundamental work on hip arthroplasty has been reported by Charnley. ${ }^{7} \mathrm{He}$ described removal of the greater trochanter with its attached gluteal muscles in the approach to the hip. Reattachment of this bony fragment and maintenance of its position can be difficult in the rheumatoid patient because of osteoporosis (fig 3), and non-union rates of $20 \%$ have been reported. ${ }^{89}$ Because of these problems I prefer to avoid the trochanteric approach unless I require the excellent exposure it affords.

Other approaches to the hip employ posterior or anterolateral routes and their use depends almost entirely on the surgeon's preference. By avoiding trochanteric detachment they permit more rapid mobilisation of the patient, though they may make positioning of the prosthetic components a little more difficult.

\section{Prosthetic fixation}

Non-cemented hip arthroplasties have attracted much interest over recent years, but rheumatoid

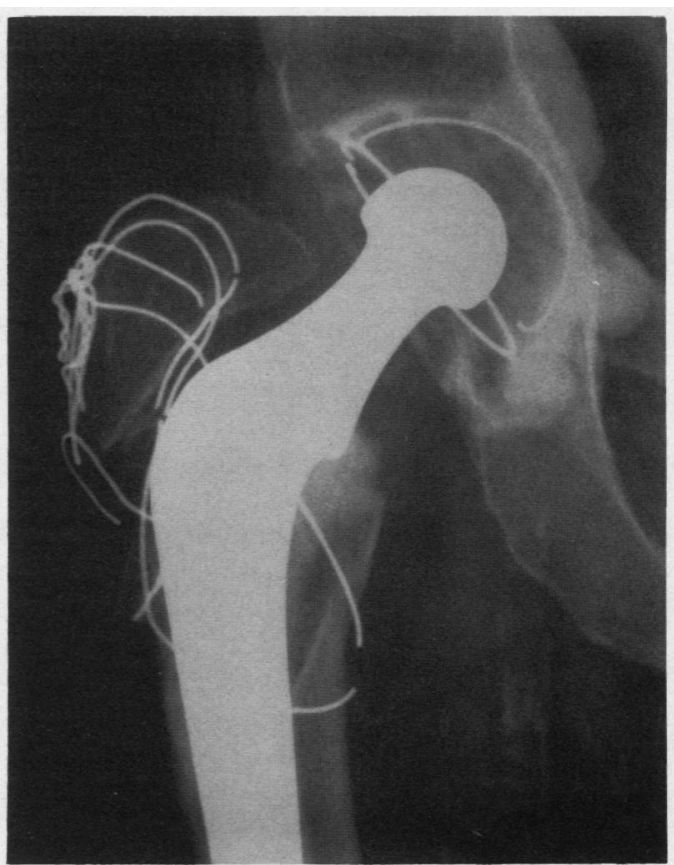

Figure 3 Charmley low friction arthroplasty with non-union of the greater trochanteric fragment and fracture of the wires.

arthritis, where there is often osteoporosis and an associated wide femoral medullary cavity, remains a relative contraindication for this method. The use of methylmethacrylate bone cement remains the method of choice in the rheumatoid patient.

\section{Intraoperative problems}

The effect of rheumatoid disease upon the bone and joint and the patterns of destruction produced predispose to the occurrence of several intraoperative problems.

\section{FEMORAL SHAFT FRACTURE}

Dislocation of the hip may be difficult, especially if there is protrusio acetabuli, and this may result in a considerable stress being put upon the osteoporotic femoral shaft, which may fracture as a result. If the hip cannot be dislocated despite removing the capsule and the anterior and superior margins of the acetabulum the femoral neck should be sectioned in situ and the head removed from the acetabulum separately.

\section{STEM PERFORATION}

It is the osteoporotic bone of the femur which again makes this complication more likely than in osteoarthritis, and it is not always easy to recognise intraoperatively. Patients with juvenile chronic arthritis will have narrow medullary cavities necessitating the use of special prostheses, and perforation may be difficult to avoid. This type of femur is probably best prepared by using powered reamers which are directed by a blunt guide wire using $x$ ray control if this is available. Perforation of the shaft can result in persistent postoperative pain and contribute towards early loosening. ${ }^{10}$ 


\section{PROTRUSIO ACETABULI}

An acetabular cup allowed to sit in an acetabulum where there is protrusio will lie in a superior position and will have little or no support from the thin or deficient medial wall. The surgeon's aim must be to provide support for the cup to sit in as near to an anatomical position as possible.

The medial acetabular wall may be reinforced by bone cement, bone, wire mesh, or support rings which bear on the acetabular rim. Charnley suggested that moderate degrees of protrusio with an intact medial wall might be managed by cement alone, ${ }^{11}$ and the more recent development of flanged and 'Ogee' cups facilitates this method by both sitting on the acetabular rim and providing support and pressurisation for the cement (fig 4).

More severe degrees of protrusio and the presence of a medial wall defect can necessitate the use of bone graft. At primary procedures this can be obtained by cutting the femoral neck into slices about $2 \mathrm{~mm}$ thick before section of the neck. Cement can be applied directly to the surface of the grafts. Such grafts have been shown to incorporate and remodelling of the medial wall of the acetabulum has been demonstrated. 1213

\section{Results of total hip replacement}

Hip arthroplasty gives good results in patients with rheumatoid arthritis, with excellent pain relief in over $90 \%$ of patients. The return of walking ability and other lower limb functions is more difficult to assess because the other lower limb joints are often affected. ${ }^{8}$ Simultaneous arthroplasty of both hips or both knees has been recommended in patients with multiple lower limb joint problems and good results have been reported. ${ }^{14}$ I have only limited experience of this approach but would warn that the patient must be made aware of the need for intensive and prolonged rehabilitation measures postoperatively.

Figure 4 Protrusio acetabuli treated by the use of a flanged cup to pressurise the cement mantle. Note the escape of the osteoporotic greater trochanter from the intact wires.
Postoperative rehabilitation usually presents few problems, though associated arm joint disease may make the use of walking aids difficult. Mobilisation can usually be started on the third or fourth day after the operation and in most cases the patient will be discharged from hospital within two weeks of surgery.

\section{Complications}

ANAEMIA

A low haemoglobin concentration is 'normal' for many rheumatoid patients and on occasion even the moderate blood loss to be expected during hip arthroplasty can lower it to such an extent that symptoms ensue. This can have an adverse effect on recovery from the operation both in terms of mobilisation and wound healing, and transfusion may be required to restore the haemoglobin to the 'normal' preoperative concentration.

\section{DEEP VENOUS THROMBOSIS}

Deep venous thrombosis is a common complication of hip arthroplasty with an overall incidence in excess of $50 \%$ and an incidence of pulmonary embolism of $6 \% .{ }^{15}$ Patients with rheumatoid arthritis are said to have a low risk of thromboembolic disease, however. ${ }^{16}$ Avoiding the trochanteric approach to the hip is also said to be beneficial, and there is some evidence that the use of spinal rather than general anaesthesia will halve the incidence of major thrombi.

Although venous thrombosis itself can have serious after effects, the low incidence of fatal pulmonary embolism (about 1\%) and the problems of haematoma and wound infection encountered during the routine use of anticoagulant drugs ${ }^{17} 18$ make it difficult to recommend a regimen of routine drug prophylaxis. Hydroxychloroquine has been reported to be effective and to have no serious side effects, ${ }^{19}$ though patients not infrequently experience short lived nausea. The results of a large double blind study of this agent are awaited. Graded compression stockings have also been shown to be of value ${ }^{20}$ and are widely used, and intraoperative calf compression also has its advocates. Whatever method is used early mobilisation is essential.

\section{INFECTION}

Infection, especially deep infection, is usually incompatible with survival of the arthroplasty, and deep infection occurs four times more commonly in rheumatoid arthritis than in osteoarthritis. ${ }^{21}$ This is usually a late event and suggests haematogenous infection from a distant site such as a skin ulcer. The use of systemic steroids also increases the risk of infection. ${ }^{22}$

Antibiotic prophylaxis is now almost universal with arthroplasties, and it has been suggested that this eliminates any differences between rheumatoid and osteoarthritic patients in the early postoperative period..$^{23}$ Because of the risk of haematogenous infection prophylactic antibiotics should be used to cover any episode 
of infection at any time postoperatively and all patients should be screened for the presence of occult infection, especially in the urinary tract, preoperatively.

\section{LOOSENING}

Loosening may be purely mechanical or have an infective cause; differentiating the two can be very difficult. In infective loosening the organism is often of low pathogenicity (Staphylococcus epidermidis or diphtheroids), there will be no clinical signs of infection around the hip, there may be no leucocytosis, and the sedimentation rate or $\mathrm{C}$ reactive protein concentration, or both, may be difficult to interpret. Radiographs will show lucent lines at the bonecement junction around the prosthesis and there may be endosteal erosions (fig 5). These signs can be present without infection, however. Bone scanning with both technetium-99m and gallium-67 citrate can be very helpful because, although both types of loosening will show an increased uptake of technetium, only infected hips will show an increased uptake of gallium. ${ }^{24}$ This method is not infallible, however, and aspiration of the hip on one or more occasions with the use of special bacteriological techniques should be used before carrying out any revision surgery. The organisms with low pathogenicity and even Staphylococcus aureus can usually be controlled sufficiently with systemic antibiotics and antibiotic loaded bone cement, making revision of the arthroplasty to another hip replacement possible in one or two stages provided that the bone stock is sufficient. Gram negative bacteria and other more difficult organisms are usually incompatible with this

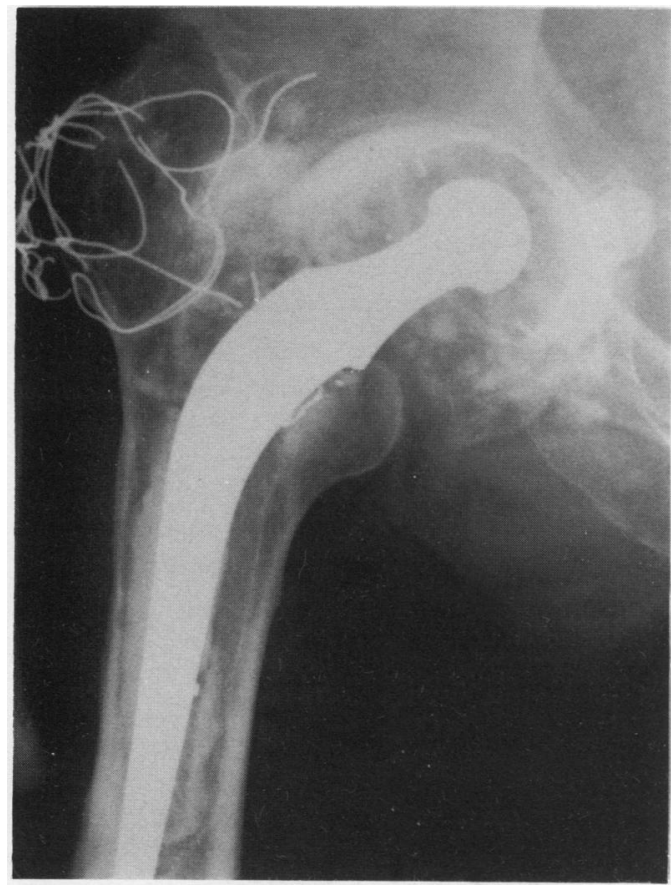

Figure 5 Gross loosening of both components of the arthroplasty. The femoral component has subsided into the femoral canal with resorption of the bone from the calcar. Note the lucency between the cement and bone and the endosteal erosions, which are most marked medially. The acetabular component has migrated into the pelvis. Once again note the radiolucent zone between the cement and the bone. method of management and removal of the arthroplasty is usually necessary (see below).

Mechanical loosening is often thought to be more prevalent in rheumatoid patients because of the poor quality of the bone and the common acetabular abnormalities. Beddow, however, considers that this is not proved and suggests that the lighter frame and the limited activities of most of these patients counter the potential problems with prosthetic fixation. ${ }^{25}$ Studies of younger patients in a variety of diagnostic groups ${ }^{46}$ suggest that aseptic loosening may occur in as many as $57 \%$ by five years after operation. Young patients with rheumatoid arthritis had a better prognosis than other groups, again probably because of their limited activities.

\section{Excision arthroplasty of the hip}

Excision arthroplasty ${ }^{27}$ has little place in the primary management of the rheumatoid hip, though it can be useful for the relief of pain and the correction of severe deformity in a chairbound patient. It has a role as a salvage procedure for failed arthroplasty when reimplantation cannot be considered because of the nature of the infecting organism or the lack of bone stock. It will provide good pain relief but, because of the resulting instability and shortening, walking is likely to be difficult even with the use of a shoe raise and a walking aid.

\section{Conclusion}

The excellent pain relief and the good long term results make total hip replacement a 'winner' in the surgical management of rheumatoid arthritis. No other surgical procedure on the hip can match the results it achieves.

1 Duthie R B, Harris C M. A radiographic and clinical survey of the hip joint in seropositive rheumatoid arthritis. Acta of the hip joint in seropositive rhe

2 Hastings D E, Parker S M. Protrusio acetabuli in rheumatoid arthritis. Clin Orthop 1975; 108: 76-83.

3 Welch R B, Charnley J. Low friction arthroplasty of the hip in rheumatoid arthritis and ankylosing spondylitis. Clin Orthop 1970; 72: 22-32.

4 Dorr L D, Takei G K, Conaty J P. Total hip arthroplasties in patients less than forty five years old. $\mathcal{f}$ Bone foint Surg $[A m]$ 1983; 65: 474-9.

5 Conaty J P. Surgery of the hip and knee in patients with rheumatoid arthritis. F Bone foint Surg [Am] 1973; 55: 301-14.

6 Mogenson B, Brattstrom H, Ekelund L, Svantesson H, Lidgren L. Synovectomy of the hip in juvenile chronic arthritis. F Bone foint Surg [Br] 1982; 64: 295-9.

7 Charnley J. Low friction arthroplasty of the hip: theory and practice. Berlin: Springer, 1979.

8 Colville J, Raunio P. Charnley low friction arthroplasties of the hip in rheumatoid arthritis. $\mathcal{f}$ Bone foint Surg $[B r]$ 1978; 60: 498-503.

9 Ranawat C S, Dorr L D, Inglis A E. Total hip arthroplasty in protrusio acetabuli of rheumatoid arthritis. $\mathcal{J}$ Bone foint Surg [Am] 1980; 62: 1059-64.

10 Talab Y A, States J D, Evarts C Mc C. Femoral shaft perforation: a complication of total hip reconstruction. Clin Orthop 1979; 141: 158-65.

11 Sotelo-Garza A, Charnley J. The results of Charnley arthroplasty of the hip performed for protrusio acetabuli. Clin Orthop 1978; 132: 12-18.

12 McCallum D E, Nunley J A, Harrelson J M. Bone grafting in total hip replacement for acetabular protrusion. $\mathcal{f}$ Bone total hip replacement for acetabular

13 Mayer G, Hartsell $\mathrm{K}$. Hip replacement in acetabular protrusion. Acta Orthop Scand 1985; 56: 461-3.

$14 \mathrm{McDonald} I$. Bilateral replacement of the hip and knee in rheumatoid arthritis. $\mathcal{F}$ Bome foint Surg [Br] 1982;64: rheum 8 .

15 Sikorski J M. Thromboembolic complications. In: Ling R S M, ed. Complications of total hip replacement. Edinburgh: Churchill Livingstone, 1984. 
16 Sikorski J M, Hampson W G, Staddon G E. The natural history and aetiology of deep vein thrombosis after total hip replacement. F Bone foint Surg [Br] 1981; 63: 171-7.

17 Harris W H, Salzman E W, Athanasoulis C, Waltman A C, Baum S, De Saudis $R$ W. Comparison of warfarin, low molecular weight dextran, aspirin and subcutaneous heparin in prevention of venous thromboembolism following total hip replacement. If Bone foint Surg [Am] 1974; 56: 1552-62.

18 Hull R D, Raskob G E. Prophylaxis of venous embolic disease following hip and knee surgery. $\mathcal{f}$ Bone foint Surg [Am] 1986; 68: 146-50.

19 Johnson R, Loudon J R. Hydroxychloroquine prophylaxis for pulmonary embolus for patients with low friction arthroplasty. Clin Orthop 1986; 211: 151-3.

20 Ishak M A, Morley K D. Deep venous thrombosis after total hip arthroplasty: a prospective controlled trial to determine the prophylactic effect of graded pressure stockings. $\mathrm{Br} \mathcal{F}$ Surg 1981; 68: 429-32.
21 Fitzgerald R H, Nolan D R, Ilstrup D M. Deep wound sepsis following total hip arthroplasty. $f$ Bone foint Surg [Am] 1977; 59: 847-55.

22 Nixon J E. Failure patterns after total hip replacement. $B$ Med F 1983; 286: 166-70.

23 Poss R, Ewald F C, Thomas W H, Sledge C B. Complications of total hip replacement arthroplasty in patients with rheumatoid arthritis. F Bone foint Surg [Am] 1976; 58: rheumatoid

24 Klenerman $L$. The management of the infected prosthesis. f Bone foint Surg [Br] 1984; 66: 645-51.

25 Beddow F H. Surgical management of rheumatoid arthritis. London: Butterworth, 1988 .

26 Chandler H P, Reineck E T, Wixson R L, McCarthy J C. Total hip replacement in patients younger than thirty years old. F Bone foint Surg [Am] 1981; 63: 1426-34.

27 Girdlestone G R. Arthrodesis and other operations for tuberculosis of the hip. F Bone foint Surg (Birthday Volume) 1928. 